\title{
Optical Alignment of the High-Precision UV Spectro-Polarimeter (CLASP2)
}

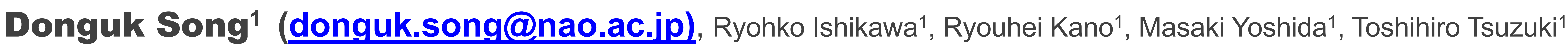
Kazuya Shinoda1, Hirohisa, Hara1, Takenori J. Okamoto', Federic Auchere², David E. Mckenzie ${ }^{3}$, Laure A. Rachmele ${ }^{3}$, Javier Trujillo Bueno ${ }^{4}$ National Astronomical Observatory of Japan, Japan; 2 Institut d'astrophysique spatiale; ${ }^{3}$ NASA Marshall Space Flight Center; ${ }^{4}$ Instituto de Astrofisica de Canarias

\section{Introduction}

\section{Chromospheric LAyer Spectro-Polarimeter (CLASP2)}

$\Delta$ is a sounding rocket experiment that is scheduled to fly in 2019

$\diamond$ aims

(1) to explore the magnetic fields in the upper chromosphere.

(2) to measure full Stokes parameters in the $\mathrm{Mg}$ II h \& $\mathrm{k}$ lines near the $280 \mathrm{~nm}$ to study wavelength-dependent variations in the polarization caused by the joint action of scattering processes and the Hanle and Zeeman effects.

In this study, we will present

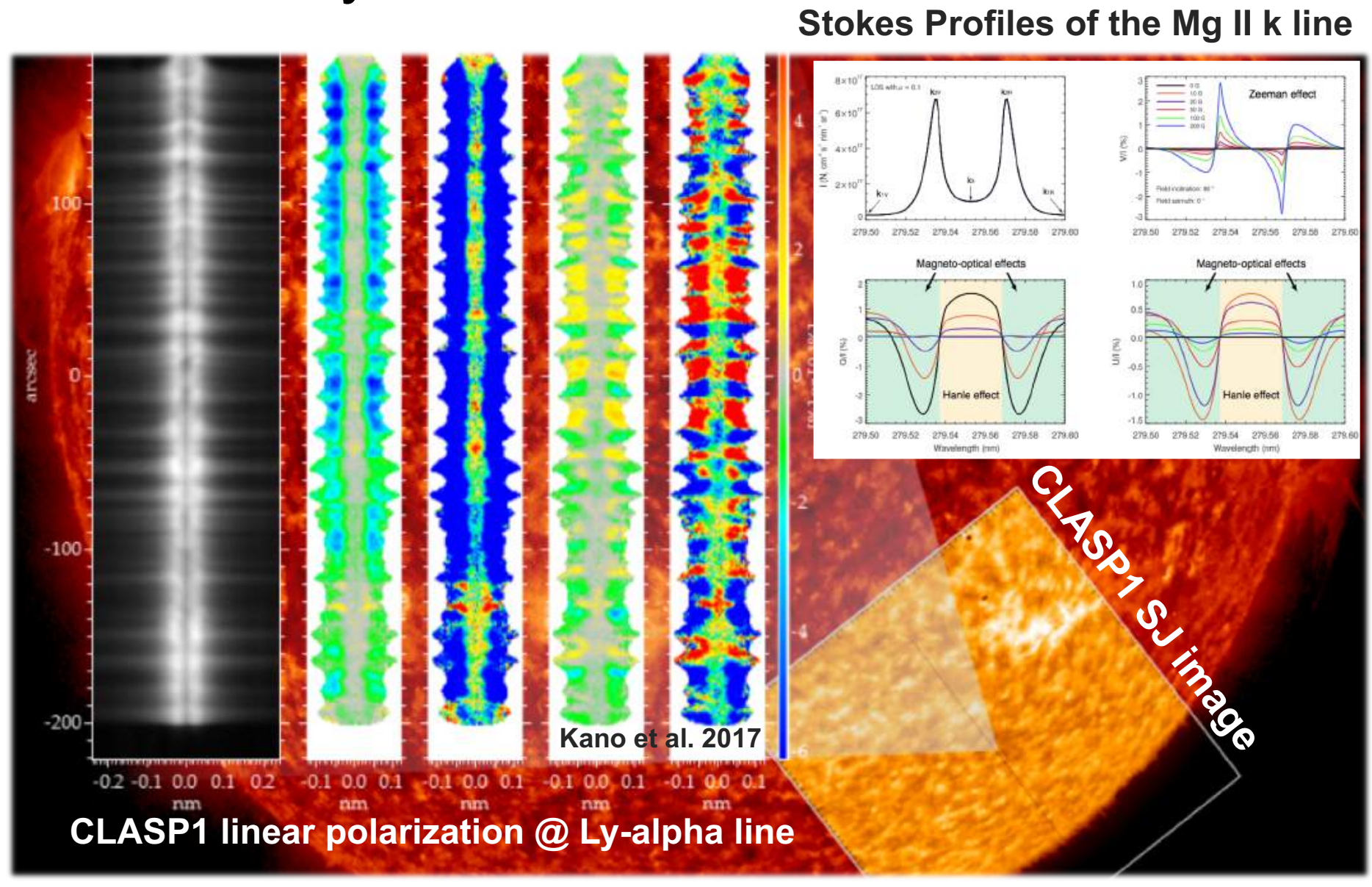

about the new optical design of the CLASP2 spectro-polarimeter.

about the method for achieving the optical alignment of the CLASP2 spectro-polarimeter.

about our results by comparing with our requirements (RMS spot radius $<13 \mu \mathrm{m}$ at the edge of the slit.).

\section{Spectro-Polarimeter}

\begin{tabular}{c|c}
\hline Spectrograph Type & Inverse Wadsworth Mounting \\
\hline Grating Type & Spherical constant-line-space grating with $1303 \mathrm{~mm}^{-1}$ groove density \\
\hline Spectral Window & $\mathrm{Mg} \mathrm{II} \mathrm{k}(279.64 \mathrm{~nm}) \& \mathrm{~h}(280.35 \mathrm{~nm})$ lines \\
\hline Plate scale & $0.55 \mathrm{arcsec} /$ pixel (spatial) $\& 0.005 \mathrm{~nm} /$ pixel (spectral) \\
\hline
\end{tabular}

\section{Optical Design of the Spectro-polarimeter (SP)}

The CLASP2 SP follows very successful design concept of the CLASP1.

- It is composed of two channels that are optically symmetric.

- It allows to measure the orthogonal states of polarizations by using the $\pm 1^{\text {st }}$ order beams diffracted by a spherical grating, simultaneously.

-The CLASP2 SP was refurbished with the minimal modification from the CLASP1 instrument.

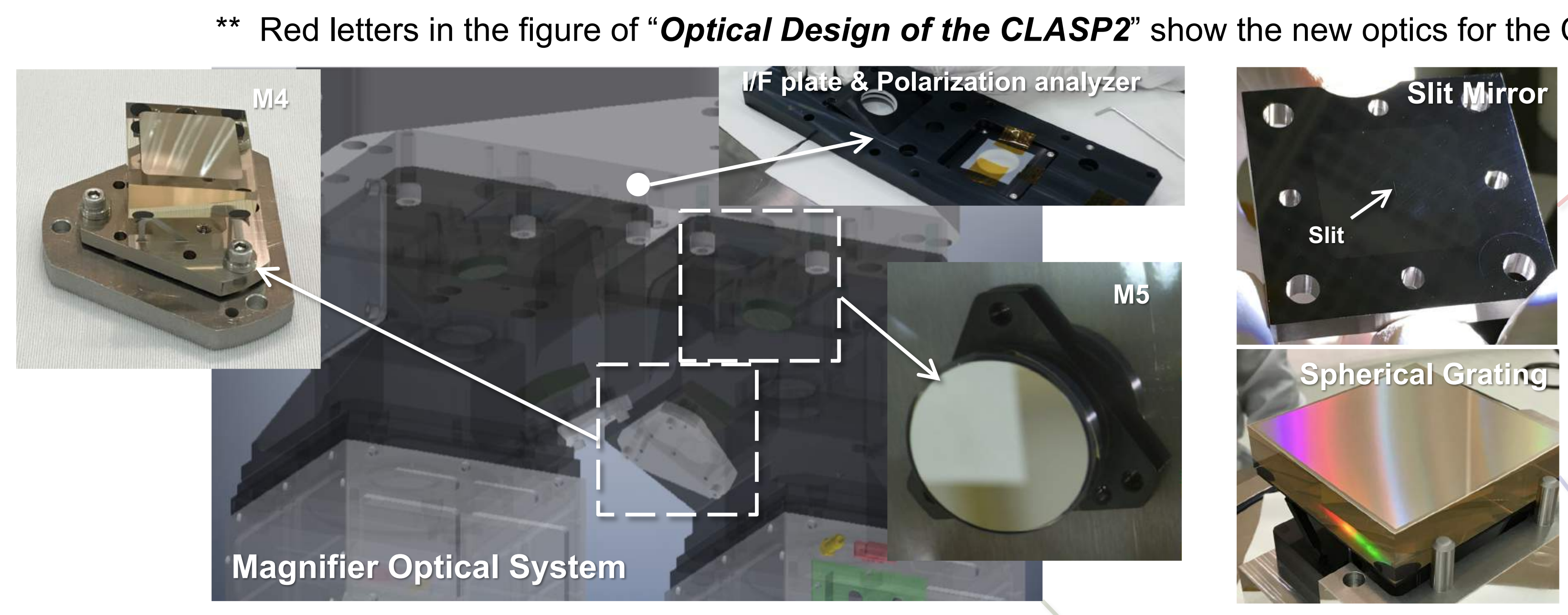

: The magnifier optical system can effectively double the focal ratio of the CLASP2 SP, which enables to keep the wavelength resolution with the CLASP1.

The magnifier optical system include the new mirrors of an off-axis hyperbolic mirror (M4) and a fold mirror (M5).

The newly fabricated mirror (M4 \& M5) were applied to the $A l+M F_{2}$ coating to improve the reflectivity over the wavelength range of $280 \pm 1 \mathrm{~nm}$

- After coating, we performed the reflectivity measurement of their witness samples $\begin{array}{lll}276 & 280 & 284 \\ \text { Wavelength }[\mathrm{mm}] & \end{array}$

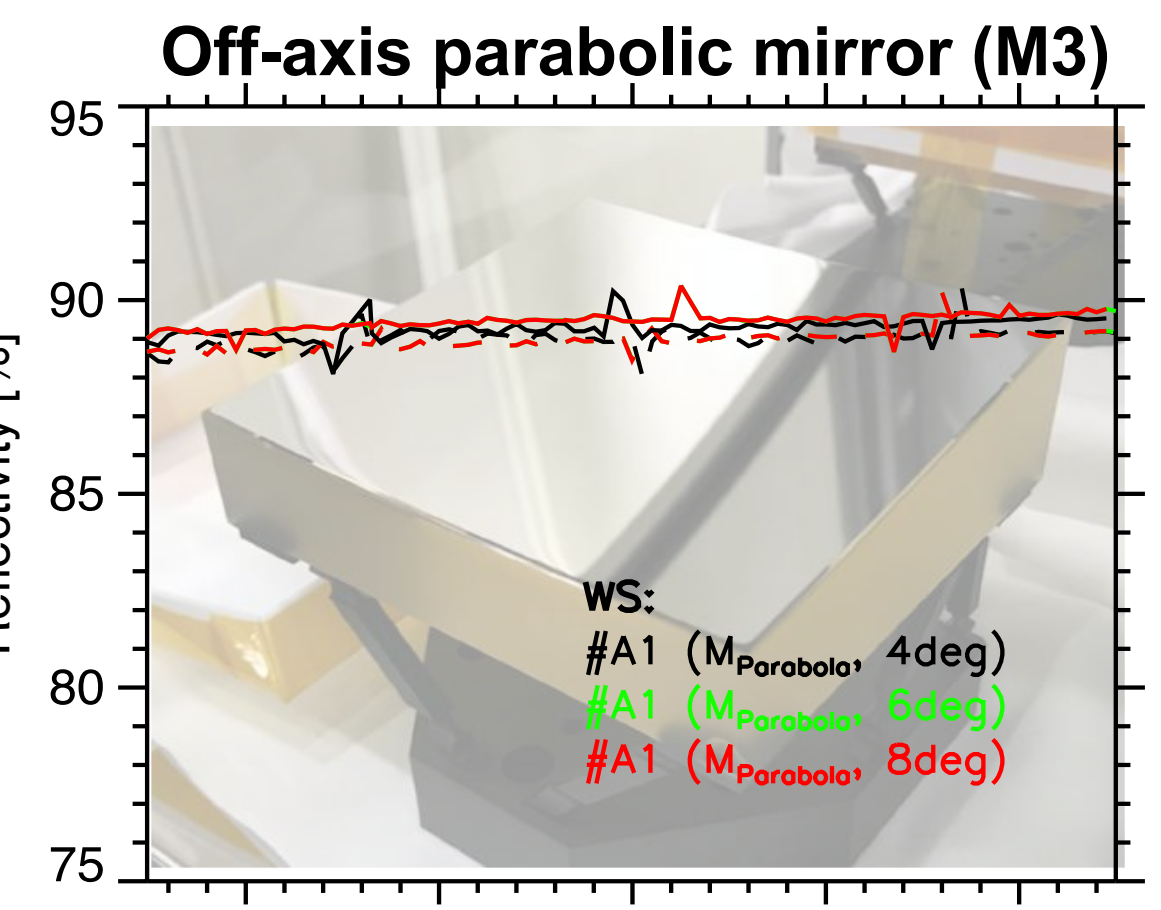

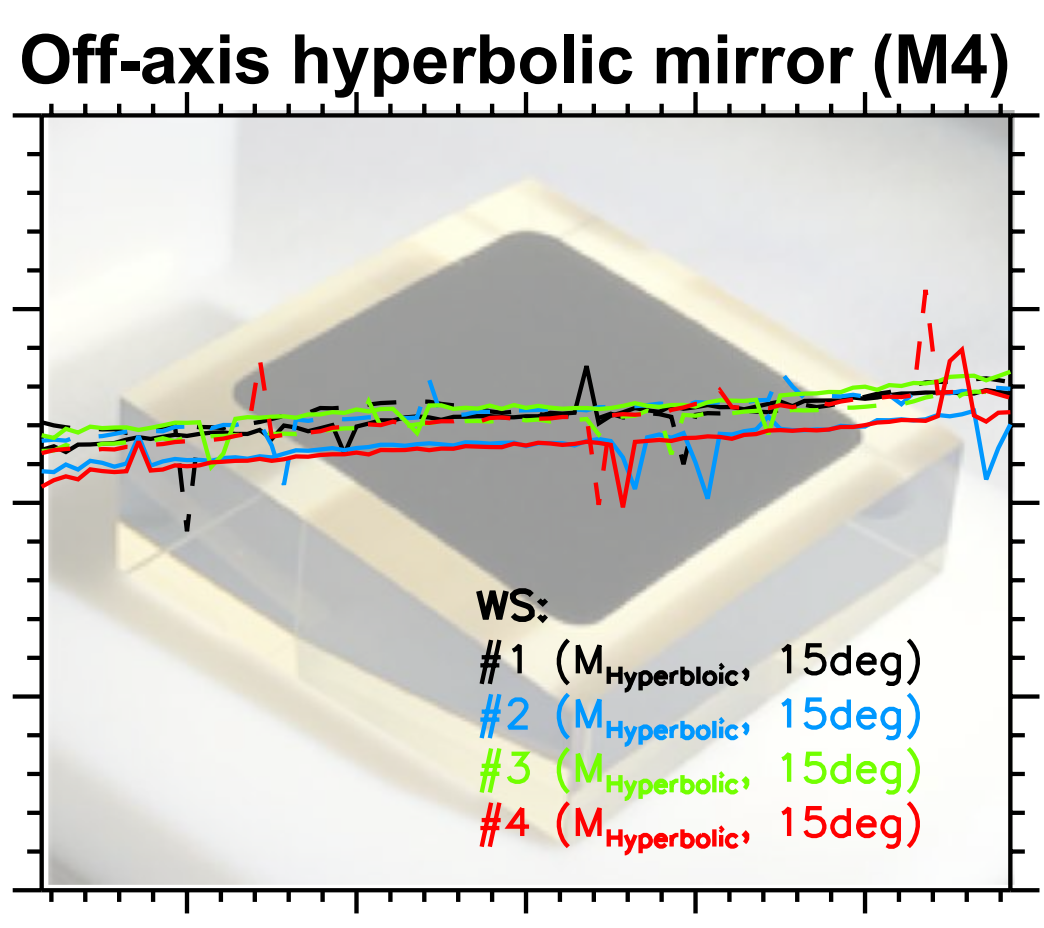

$266 \quad 273 \quad 280287 \quad 294$
Fold mirror (M5)

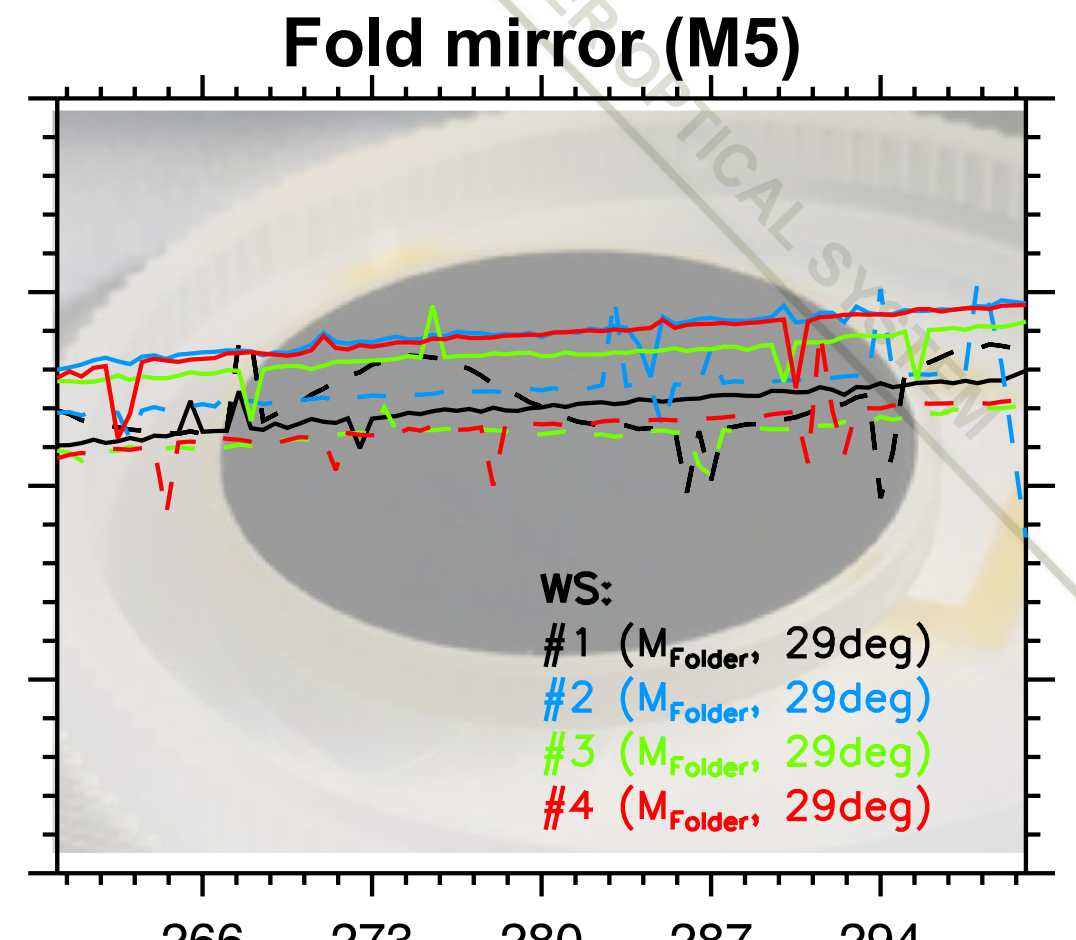

(WS) of the off-axis parabolic mirror (left), the off-axis convex hyperbolic mirror (middle), and the fold mirror (right). The WSs are 1 -inch flat mirrors that were simultaneously coated with the flight mirrors during the coating processes. The dashed and solid lines represent $\mathrm{p}$ - and s- polarized light.

- The measured reflectivity of all witness samples is larger than $85 \%$ at the predetermined Angle of Incidence (AOI).

Our results shows that it satisfies our required specification ( $>80 \%$ near the $280 \mathrm{~nm}$ ) of the SP mirrors.
Optical Design of the CLASP2

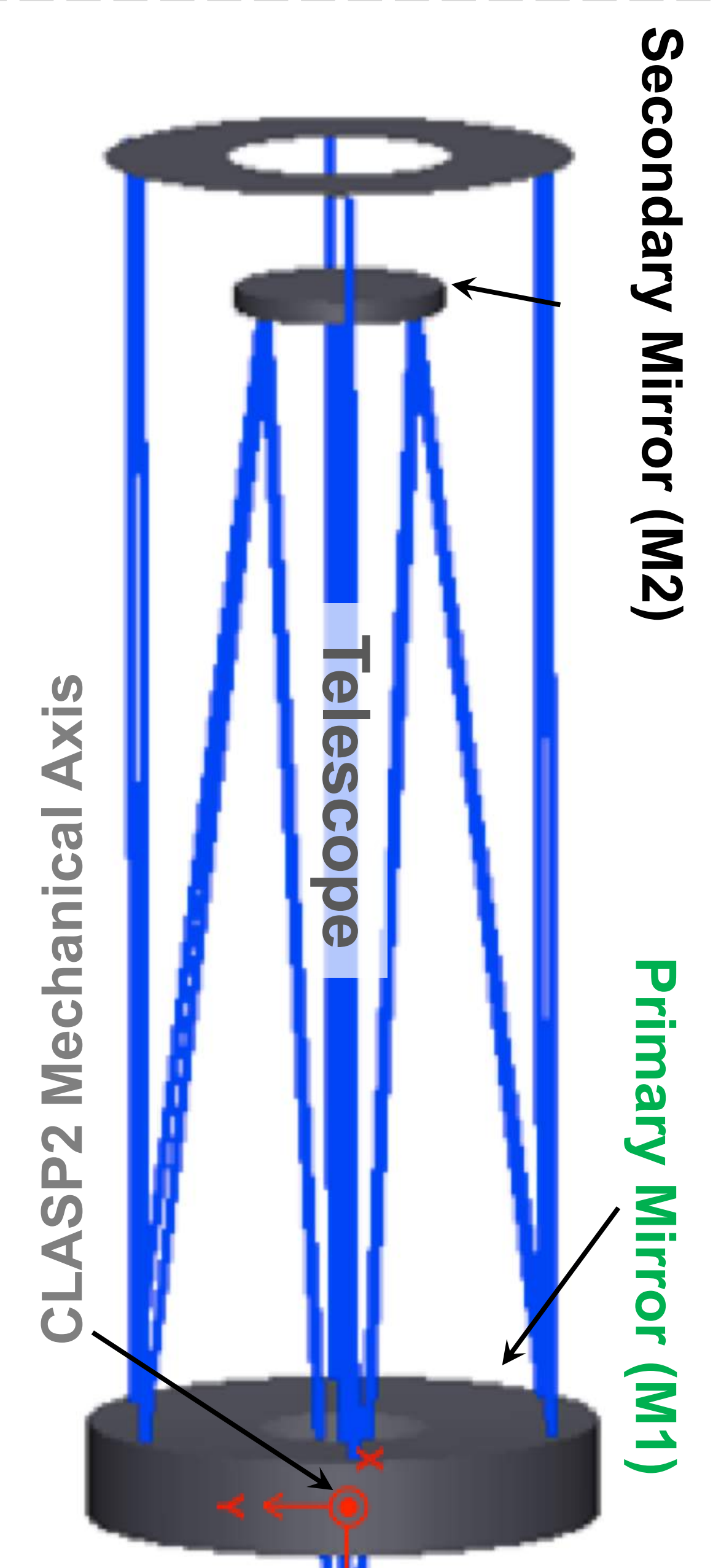

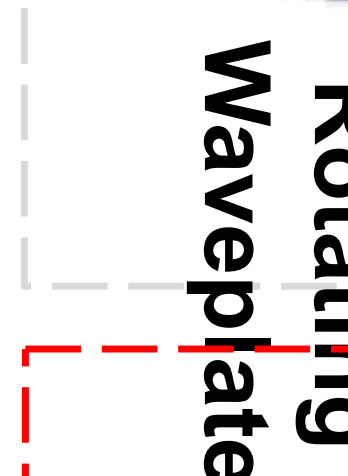
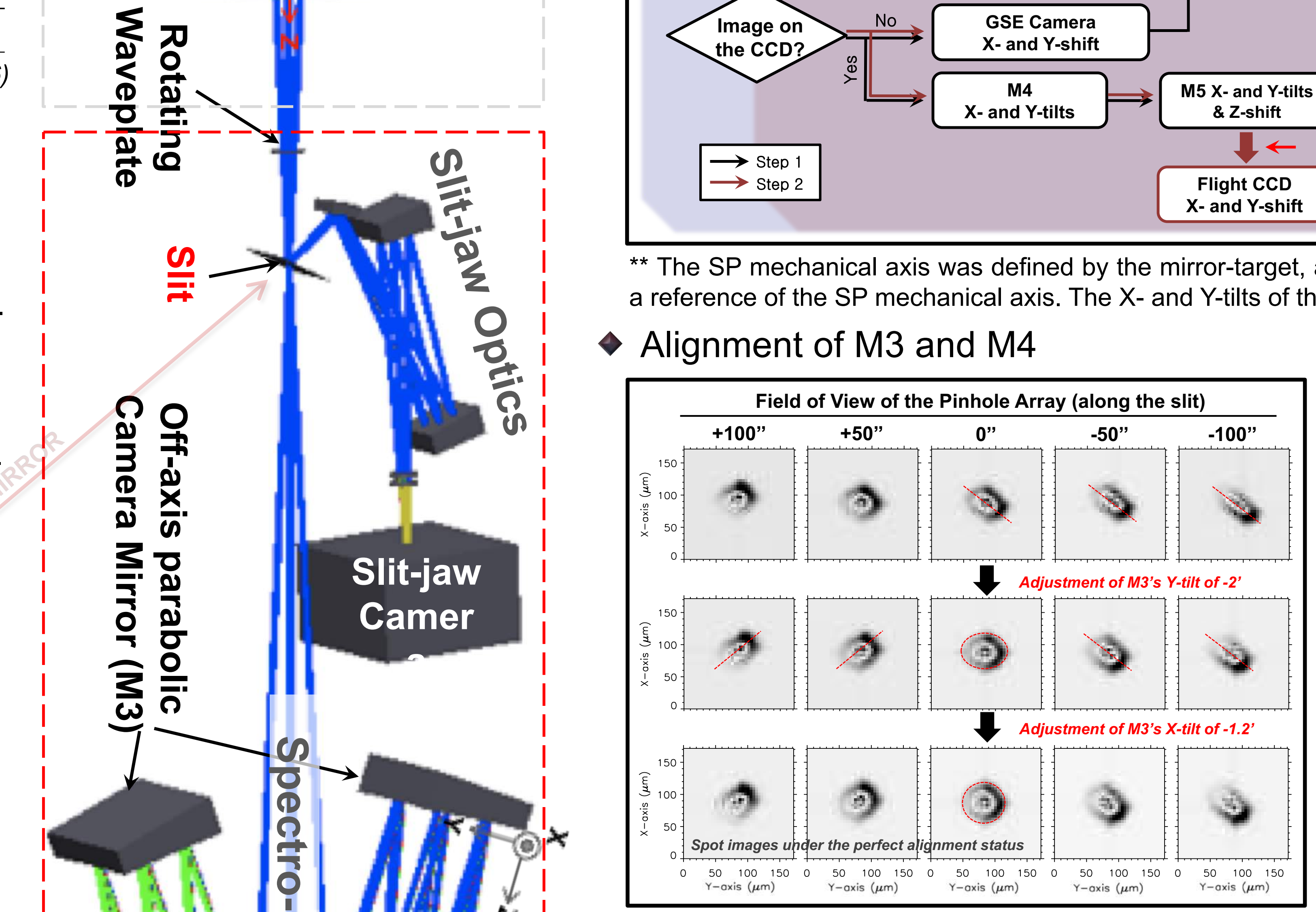

Alignment of M5

- The adjustment of M5's $X$ - and $Y$-tilts induces the inthe image shift along $Y$ - and $X$-directions expresses as,

$X$ image shift $(\mu m)=\frac{M 5^{\prime} s \text { Y tilt }(1)}{0.14}$

$Y$ image shift $(\mu m)=-\frac{M 5^{\prime} s X \text { tilt }(1)}{0.013}$ We investigated the RMS spot
the $2 \mathrm{D}$ Gaussian function.

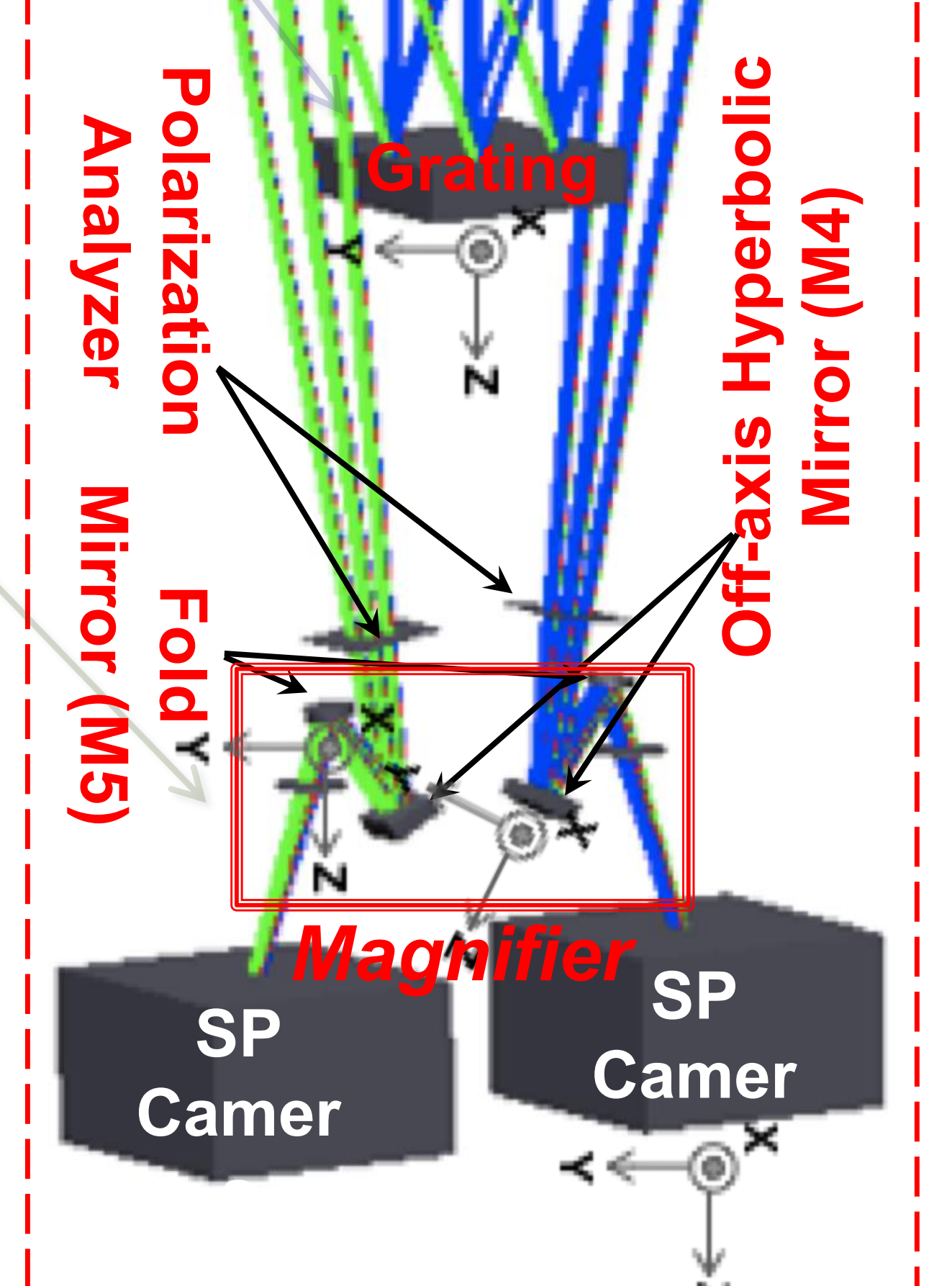

\section{Summary and Discussion}

- We established and performed an efficient optical alignment procedure for the CLASP2 SP. the slit.

"The RMS spot radius measured by $2 \mathrm{D}$ Gaussian function is measured overestimated compared to the real RMS spot radius. In

-Therefore, the SP alignment is succeeded to satisfy our requirement $(<13 \mu$ mat

Even though, we achieved a satisfactory RMS spot radius, one may wonder

(1) why M4 has an unexpectedly larger tilts

$>>$ We found image shift after $M 3$ alignment (The adjustment of $X$ - and $Y$-tils
in $Y$-and $X$-image shift). Such an image shift can introduce the M4's large tilts.

(2) why the Mg II image quality deteriorates after VL alignment.

>> From the PSF (right figure) constructed by the optical simulation,
disk size of the He-Ne line is twice larger than that of the Mg II line. 